\title{
Spin-3/2 Pentaquark Resonance Signature
}

\author{
B. G. Lasscock*', J. Hedditch, D. B. Leinweber, A. G. Williams \\ Special Research Centre for the Subatomic Structure of Matter, and Department of Physics, \\ University of Adelaide, Adelaide SA 5005, Australia \\ E-mail: blasscoc@physics.adelaide.edu.au
}

\section{W. Kamleh}

School of Mathematics, Trinity College, Dublin 2, Ireland

\section{W. Melnitchouk, A. W. Thomas, R. D. Young}

Jefferson Lab, 12000 Jefferson Ave., Newport News, VA 23606 USA

\section{J. M. Zanotti}

John von Neumann-Institut für Computing NIC/DESY

\begin{abstract}
We search for the standard lattice resonance signature of attraction between the resonance constituents which leads to a bound state at quark masses near the physical regime. We study a variety of spin- $\frac{1}{2}$ interpolators and for the first time, interpolators providing access to spin- $\frac{3}{2}$ pentaquark states. In looking for evidence of binding, a precise determination of the mass splitting between the pentaquark state and its lowest-lying decay channel is performed by constructing the effective mass splitting from the various two-point correlation functions. While the binding of the pentaquark state is not a requirement, the observation of such binding would provide compelling evidence for the existence of the $\Theta^{+}$pentaquark resonance. Evidence of binding is observed in the isoscalar spin- $\frac{3}{2}$ positive parity channel, making it an interesting state for further research.
\end{abstract}

XXIIIrd International Symposium on Lattice Field Theory

25-30 July 2005

Trinity College, Dublin, Ireland

\footnotetext{
*Speaker.

$\dagger$ Thanks to the Australian partnership for advanced computing (APAC) national facility for the computer time that made this study possible, and for further support from the South Australian partnership for advanced computing (SAPAC).
} 


\section{Introduction}

We present the results of our search for the exotic $\Theta^{+}$pentaquark resonance, which has strangeness +1 and minimal quark content $u u d d \bar{s}$. Since the spin, parity and isospin of the $\Theta^{+}$ remain open questions, we have explored $[1,2]$ a wide space of quantum numbers using an exhaustive array of interpolating fields, including for the first time a study of the $\Theta^{+}$as a possible spin-3/2 state [2]. Key to this work is the formulation of a robust resonance signature in lattice QCD that can distinguish a resonance from possible scattering states. The volume dependence of the residue of the lowest lying state has been proposed as a way to identify a resonance [3, 4]. Alternatively, hybrid boundary conditions, i.e. choosing a different boundary condition for the $u, d$-quarks compared to the $s$-quark, have been proposed in Refs. $[5,6,7]$ to differentiate the resonance from a scattering state. Our method, which is complementary to these approaches, is to look for sufficient attraction between the constituents of the pentaquark state such that the mass of the pentaquark state is less than the energy of the corresponding free decay channel. We refer to this in $[1,2]$ as the "standard lattice resonance signature" in lattice QCD because we have universally observed this behaviour at the quark masses that have been considered in studies of conventional baryons $[8,9,10,11,12]$.

In Sec. 2 we present the details of our lattice simulation. In Sec. 3 we present our analysis of the even and odd parity, spin-1/2 and spin-3/2 pentaquark states, in both the isoscalar and isovector channels. As we will see there is clear evidence of the standard lattice resonance signature in the $I\left(J^{P}\right)=0\left(3 / 2^{+}\right)$channel. As we conclude in Sec. 4 , this $0\left(3 / 2^{+}\right)$state is a worthy candidate for future study of the $\Theta^{+}$pentaquark in lattice QCD.

\section{Lattice Details}

\subsection{Lattice Simulations}

In our analysis we use a large $20^{3} \times 40$ lattice. Using the mean-field $\mathscr{O}\left(a^{2}\right)$-improved LuscherWeisz plaquette plus rectangle action [13], the gauge configurations are generated via the CabibboMarinari pseudoheat-bath algorithm with three diagonal SU(2) subgroups looped over twice. The lattice spacing is $0.128(2) \mathrm{fm}$, determined using the Sommer scale $r_{0}=0.49 \mathrm{fm}$. For the fermion propagators, we use the FLIC fermion action [14], an $\mathscr{O}(a)$-improved fermion action with excellent scaling properties providing near continuum results at finite lattice spacing [15]. A fixed boundary condition in the time direction is implemented by setting $U_{t}\left(\vec{x}, N_{t}\right)=0 \forall \vec{x}$ in the hopping terms of the fermion action, and periodic boundary conditions are imposed in the spatial directions. Gaugeinvariant Gaussian smearing [16] in the spatial dimensions is applied at the fermion source at $t=8$ to increase the overlap of the interpolating operators with the ground states. Six quark masses are used in the calculations, with $\kappa=\{0.12780,0.12830,0.12885,0.12940,0.12990,0.13025\}$ providing $a m_{\pi}=\{0.540,0.500,0.453,0.400,0.345,0.300\}$. The strange quark mass is taken to be the third largest $(\kappa=0.12885)$ quark mass. This $\kappa$ provides a pseudoscalar mass of $697 \mathrm{MeV}$ which compares well with the experimental value of $\sqrt{2 M_{\mathrm{K}}^{2}-M_{\pi}^{2}}=693 \mathrm{MeV}$ motivated by leading order chiral perturbation theory. The error analysis is performed by a second-order, single-elimination jackknife, with the $\chi^{2}$ per degree of freedom obtained via covariance matrix fits. Further details of the fermion action and simulation parameters are provided in Refs. [14, 15]. 


\subsection{Interpolating Fields}

In this section we review the interpolating fields that we use in our pentaquark studies. The two general types of pentaquark interpolating fields we consider are those based on an " $N K$ " configuration (either $n K^{+}$or $p K^{0}$ ), and those based on a "diquark-diquark- $\vec{s}$ " configuration. Below is a summary of the interpolating fields we use that only couple to spin-1/2 states,

$$
\begin{aligned}
\chi_{N K} & =\frac{1}{\sqrt{2}} \varepsilon^{a b c}\left(u^{T a} C \gamma_{5} d^{b}\right)\left\{u^{c}\left(\bar{s}^{e} i \gamma_{5} d^{e}\right) \mp(u \leftrightarrow d)\right\} \\
\chi_{\overparen{N K}} & =\frac{1}{\sqrt{2}} \varepsilon^{a b c}\left(u^{T a} C \gamma_{5} d^{b}\right)\left\{u^{e}\left(\bar{s}^{e} i \gamma_{5} d^{c}\right) \mp(u \leftrightarrow d)\right\} \\
\chi_{P S} & =\varepsilon^{a b c} \varepsilon^{a e f} \varepsilon^{b g h}\left(u^{T e} C d^{f}\right)\left(u^{T g} C \gamma_{5} d^{h}\right) C \bar{s}^{T c} \\
\chi_{S S} & =\frac{1}{\sqrt{2}} \varepsilon^{a b c}\left(u^{T a} C \gamma_{5} d^{b}\right)\left(u^{T c} C \gamma_{5} d^{e}\right) C \bar{s}^{T e},
\end{aligned}
$$

For $\chi_{N K}$ and $\chi_{\widetilde{N K}}$, the - and + corresponds to the isospin $I=0$ and 1 channels, while $\chi_{P S}$ and $\chi_{S S}$ access isoscalar and isovector states respectively.

In addition to these fields we have also considered an $N K^{*}$-type field that couples to both spin$1 / 2$ and spin-3/2 states and we propose to study a diquark-diquark style field, similar to that used in [7], which couples to both spin-1/2 and spin-3/2 states, including isovector states,

$$
\begin{aligned}
\chi_{N K^{*}}^{\mu} & =\frac{1}{\sqrt{2}} \varepsilon^{a b c}\left(u^{T a} C \gamma_{5} d^{b}\right)\left\{u^{c}\left(\vec{s}^{e} i \gamma^{\mu} d^{e}\right) \mp(u \leftrightarrow d)\right\} \\
\chi_{V S}^{\mu} & =\varepsilon^{a b c}\left(u^{T a} C \gamma_{5} \gamma^{\mu} d^{b}\right)\left\{\left(u^{T c} C \gamma_{5} d^{e}\right) \mp\left(u^{T e} C \gamma_{5} d^{c}\right)\right\} C \vec{s}^{e T},
\end{aligned}
$$

where the - and + corresponds to the isospin $I=0$ and 1 channels, respectively. To project states of definite spin from the correlation function we apply the spin projection operators [10],

$$
\begin{aligned}
& P_{\mu v}^{\frac{3}{2}}(p)=g_{\mu v}-\frac{1}{3} \gamma_{\mu} \gamma_{v}-\frac{1}{3 p^{2}}\left(\gamma \cdot p \gamma_{\mu} p_{v}+p_{\mu} \gamma_{v} \gamma \cdot p\right), \\
& P_{\mu v}^{\frac{1}{2}}(p)=g_{\mu v}-P_{\mu \nu}^{\frac{3}{2}}(p) .
\end{aligned}
$$

\subsection{Lattice Resonance Signature}

The presence of binding at quark masses near the physical regime has been universally observed in our studies of nucleon resonances and is central to the study of the electromagnetic properties of decuplet baryons [17] and their transitions [18, 19, 20] in lattice QCD. In Fig. 1 we show the spectrum of nucleon and $\Delta$ resonances $[9,10]$. The solid curve is the S-Wave $N+\pi$ decay channel energy corresponding to the $1 / 2^{-}$and $3 / 2^{-}$states which clearly become bound, i.e. the mass of the resonance becomes less than its decay channel energy, at the quark masses shown. This what we refer to as the standard lattice resonance signature of binding at quark masses near the physical regime. Note that the $3 / 2^{+}$state also becomes bound as it decays to a P-Wave $N+\pi$ (not shown), which due to finite volume effects is at a higher energy than the $\mathrm{S}$-Wave $N+\pi$.

To search for evidence of the $\Theta^{+}$we require a precise measurement of the splitting between the mass of the pentaquark state and the free decay channel energy. Hence we define an effective mass splitting where both correlated and systematic errors are minimised. For example in an S-wave decay channel,

$$
\Delta M^{\mathrm{eff}}(t) \equiv M_{5 q}^{\mathrm{eff}}(t)-\left(M_{B}^{\mathrm{eff}}(t)+M_{M}^{\mathrm{eff}}(t)\right)
$$



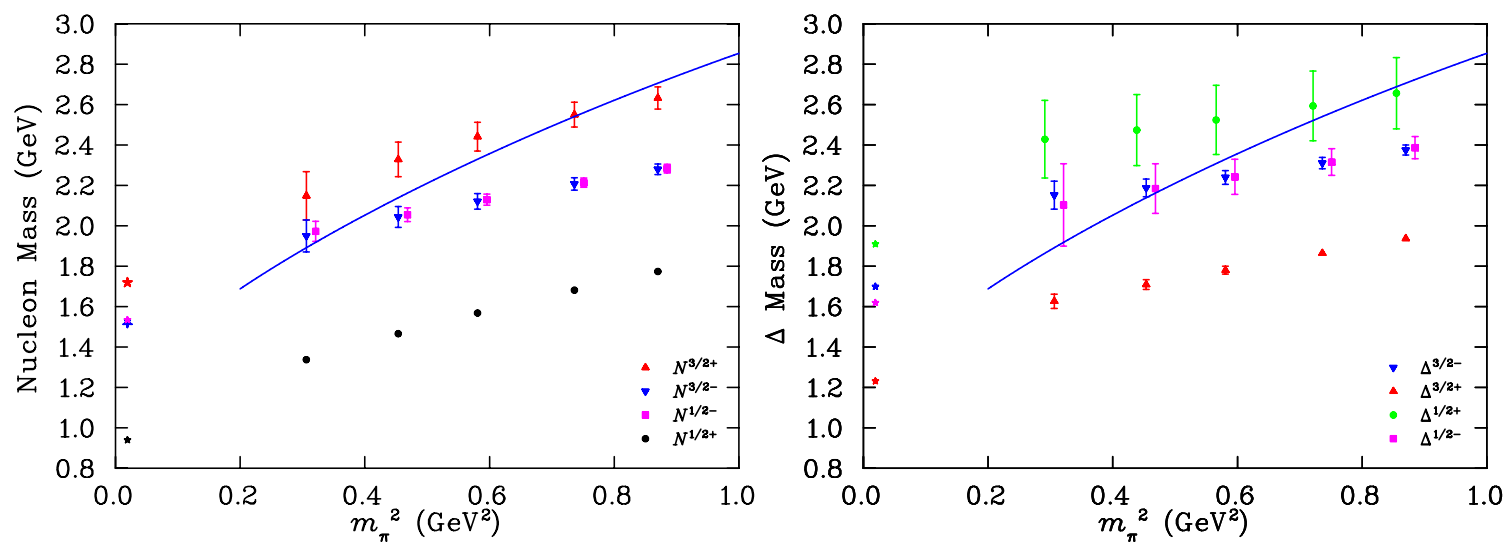

Figure 1: Summary of nucleon resonances $[9,10]$, the solid curve is the mass of the $\mathrm{S}$-Wave $N+\pi$ decay channel corresponding to $1 / 2^{-}, 3 / 2^{-}$parity states.

$$
\stackrel{t \rightarrow \infty}{=} m_{5 q}-\left(m_{B}+m_{M}\right)
$$

where $M_{B}^{\text {eff }}(t)$ and $M_{M}^{\text {eff }}(t)$ are the appropriate baryon and meson effective masses for a specific channel. In the case of a P-Wave decay channel, the effective masses of each decay constituent is combined with the minimum nontrivial momentum on the lattice, $2 \pi / L$, to create the effective energy $E^{\text {eff }}(t)=\sqrt{\left(M^{\text {eff }}(t)\right)^{2}+(2 \pi / L)^{2}}$.

\section{Results}

In this section we present a summary of the findings from our studies of the $\Theta^{+}$pentaquark. In Fig 2 we present a summary of our most relevant results. We find that the negative parity states, spin-1/2 and spin-3/2, isocalar and isovector are more massive than their respective decay channels. While this does not necessarily exclude the existence of the $\Theta^{+}$pentaquark, Mathur et al. [3] have shown that the $0\left(1 / 2^{-}\right)$is indeed a $N+K$ scattering state. Its mass is shifted higher than the free decay channel energy, due to finite volume effects, and this shift can be related to the scattering length for which we find values the order of $-1 \mathrm{fm}$ with a trend towards the experimental value. In
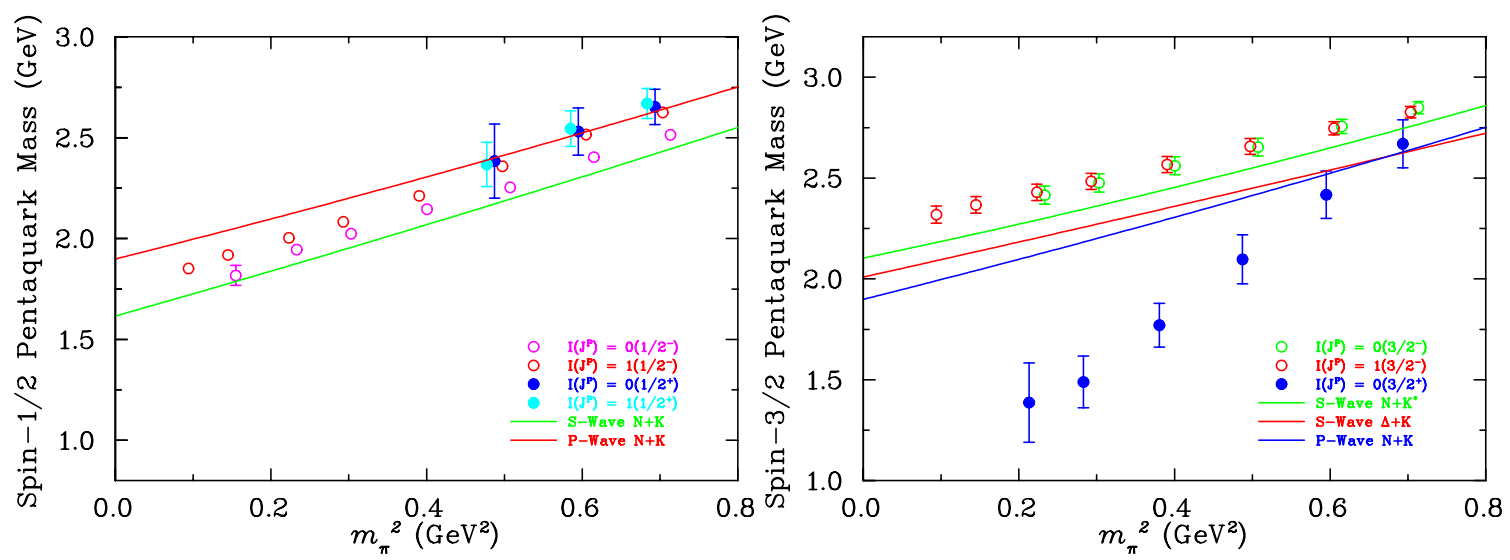

Figure 2: A summary of results extracted with spin-1/2 and spin-3/2 pentaquark interpolators. 
the spin- $1 / 2$, positive parity channel we found that the mass of the pentaquark states are consistent with their decay channel energies.

Our most striking observation of the standard lattice resonance signature is in the $0\left(3 / 2^{+}\right)$ channel, which shows evidence of binding at quark masses near the physical regime and also displays the appropriate approach to the limit of very heavy quark masses that one expects for a five quark state based on quark counting rules.
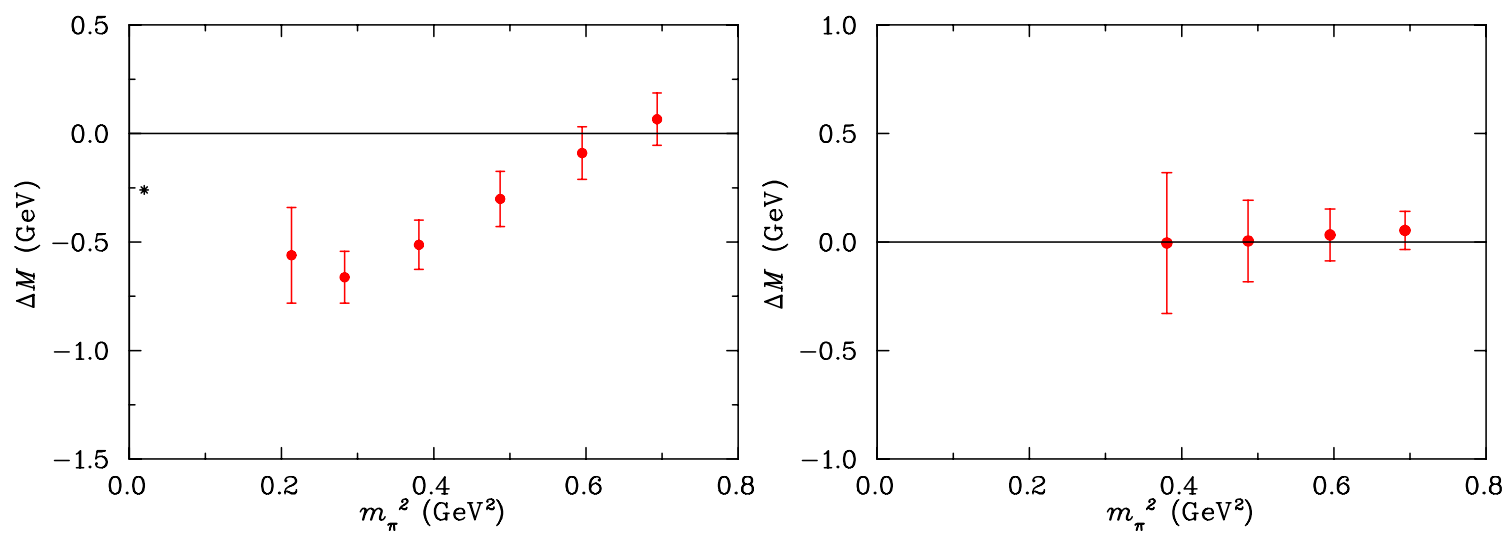

Figure 3: The masses of the $0(3 / 2+)$ (left) and $0(1 / 2+)$ (right) pentaquark states minus the P-Wave $N+K$ decay channel energy.

Figure 3 shows the masses of the $0(1 / 2+)$ and $0(3 / 2+)$ pentaquark states minus the P-Wave $N+K$ decay channel energy. The physical mass of the $\Theta^{+}$minus the physical P-Wave $N+K$ decay channel energy, adjusted for our finite volume lattice, is displayed for comparison. The standard lattice resonance signature is seen in the $0(3 / 2+)$ channel, whereas existence of binding in the $0(1 / 2+)$ channel remains inconclusive due to large statistical uncertainties.

\section{Conclusions}

We have completed a comprehensive analysis of the isospin and parity states of the spin-1/2 and spin-3/2 pentaquark. We find no evidence of the standard lattice resonance signature for negative parity spin-1/2 or spin-3/2 states. Due to large statistical errors we could not determine if there exists evidence of binding in the positive parity spin-1/2 channel. However, in our examination of $0\left(3 / 2^{+}\right)$pentaquark state we have discovered evidence of the standard lattice resonance signature making it a prime candidate for future study. An analysis of the volume dependence of this signature to determine if it suggests the existence of the $\Theta^{+}$pentaquark remains as future work.

\section{References}

[1] B. G. Lasscock et. al., Search for the pentaquark resonance signature in lattice qcd, hep-lat/0503008.

[2] B. G. Lasscock et. al., Spin 3/2 pentaquark resonance signature in lattice qcd, hep-lat/ 0504015.

[3] N. Mathur et. al., A study of pentaquarks on the lattice with overlap fermions, Phys. Rev. D70 (2004) 074508 [hep-ph/ 0406196 ]. 
[4] C. Alexandrou and A. Tsapalis, A lattice study of the pentaquark state, hep-lat/0503013.

[5] N. Ishii et. al., Penta-quark baryon in anisotropic lattice qcd, Phys. Rev. D71 (2005) 034001 [hep-lat/0408030].

[6] N. Ishii et. al., Anisotropic lattice qcd studies of penta-quark anti-decuplet, hep-1at/0 410022.

[7] N. Ishii, T. Doi, Y. Nemoto, M. Oka and H. Suganuma, Spin $3 / 2$ penta-quarks in anisotropic lattice qcd, hep-lat/0506022.

[8] D. B. Leinweber, W. Melnitchouk, D. G. Richards, A. G. Williams and J. M. Zanotti, Baryon spectroscopy in lattice qcd, Lect. Notes Phys. 663 (2005) 71-110.

[9] W. Melnitchouk et. al., Excited baryons in lattice qcd, Phys. Rev. D67 (2003) 114506 [hep-lat/0202022].

[10] CSSM Lattice Collaboration Collaboration, J. M. Zanotti et. al., Spin-3/2 nucleon and delta baryons in lattice qcd, Phys. Rev. D68 (2003) 054506 [hep-lat/ 0304001 ].

[11] S. Sasaki, T. Blum and S. Ohta, A lattice study of the nucleon excited states with domain wall fermions, Phys. Rev. D65 (2002) 074503 [hep-lat/0102010].

[12] QCDSF Collaboration, M. Gockeler et. al., Negative-parity baryon masses using an o(a)-improved fermion action, Phys. Lett. B532 (2002) 63-70 [hep-lat/ 0106022$].$

[13] M. Luscher and P. Weisz, On-shell improved lattice gauge theories, Commun. Math. Phys. 97 (1985) 59.

[14] CSSM Lattice Collaboration Collaboration, J. M. Zanotti et. al., Hadron masses from novel fat-link fermion actions, Phys. Rev. D65 (2002) 074507 [hep-lat/ 0110216 ].

[15] J. M. Zanotti, B. Lasscock, D. B. Leinweber and A. G. Williams, Scaling of flic fermions, Phys. Rev. D71 (2005) 034510 [hep-lat/ 0405015$].$

[16] S. Gusken, A study of smearing techniques for hadron correlation functions, Nucl. Phys. Proc. Suppl. 17 (1990) 361-364.

[17] D. B. Leinweber, T. Draper and R. M. Woloshyn, Decuplet baryon structure from lattice qcd, Phys. Rev. D46 (1992) 3067-3085 [hep-lat/9208025].

[18] D. B. Leinweber, T. Draper and R. M. Woloshyn, Baryon octet to decuplet electromagnetic transitions, Phys. Rev. D48 (1993) 2230-2249 [hep-lat/9212016].

[19] C. Alexandrou et. al., $N$ to delta electromagnetic transition form factors from lattice qcd, Phys. Rev. D69 (2004) 114506 [hep-lat/ 0307018 ].

[20] C. Alexandrou et. al., The $n$ to delta electromagnetic transition form factors from lattice qcd, Phys. Rev. Lett. 94 (2005) 021601 [hep-lat / 0409122 ]. 